MODELING, IDENTIFICATION AND CONTROL, 1993, VOL. 14, NO. 4, 181-191

doi:10.4173/mic.1993.4.1

\title{
Direct adaptive generalized predictive control
}

\author{
WEI WANG $\dagger$ and ROLF HENRIKSEN $\ddagger$
}

Keywords: Predictive control; adaptive control; convergence

This paper is concerned with the direct approach of adaptive generalized predictive control. An implicit model with control law parameters is developed. A direct adaptive generalized predictive control algorithm and an improved variant are suggested. Global convergence of the algorithms is analysed under some assumptions.

\section{Introduction}

Because of their flexibility and successful industrial applications, adaptive control algorithms based on generalized predictive control (GPC) or long-range predictive control strategies have recently aroused a great deal of interest in the control community (Clarke, Mohtadi and Tuffs, 1987, Keyser, De Van de Velde and Dumortier 1988, Kramer and Unbehauen 1988, Ydstie 1984, Lelic and Zarrop 1987). Nevertheless, to the best of the authors' knowledge, almost all the adaptive GPC algorithms appearing in the literature are in the indirect or explicit form, i.e. the plant parameters are estimated on-line and controller calculations are carried out based on the estimated plant parameters. The only adaptive GPC controller in direct form was reported in Ortega and Sanchez (1989) by using the assumption that the first $N_{1}\left(N_{1}\right.$ is the prediction horizon) coefficients of the plant's impulse response are known. In addition, $N_{1}$ parameter estimators were used at each sampling instant. One disadvantage of the indirect form is that the computational load is quite heavy for on-line applications, especially in adaptive GPC algorithms because multi-step predictions are involved.

In this paper the direct version of adaptive generalized predictive control is considered. A direct adaptive GPC algorithm with a priori knowledge of the first $N_{1}$ coefficients of the plant step response is suggested, but only one parameter estimator is used at each sampling instant. An improved variant without a priori knowledge of the first $N_{1}$ coefficients of the plant step response is also suggested by using a normalization technique and a parameter estimator with a dead zone.

Received 27 April 1993.

$\dagger$ Department of Automatic Control, Northeast University of Technology, Shenyang 110006, P.R. China. Currently on leave at The Norwegian Institute of Technology, Division of Engineering Cybernetics, N-7034 Trondheim, Norway.

$¥$ Norwegian Institute of Technology, Division of Engineering Cybernetics, N-7034 Trondheim, Norway.

The work was supported by the Royal Norwegian Council for Scientific and Industrial Research (NTNF) and the National Natural Science Foundation of China.

Reprinted, with permission from the American Automatic Control Council, from Proceedings of the 1992 American Control Conference, Chicago, June 24-26, 1992. 


\section{Generalized Predictive Control}

Consider a time-invariant plant described by the following CARIMA model

$$
A\left(z^{-1}\right) \Delta y(t)=B\left(z^{-1}\right) \Delta u(t-1)
$$

where $A\left(z^{-1}\right)$ and $B\left(z^{-1}\right)$ are polynomials in the backward shift operator $z^{-1}$ of the form

$$
\begin{aligned}
& A\left(z^{-1}\right)=1+a_{1} z^{-1}+\ldots+a_{n} z^{-n} \\
& B\left(z^{-1}\right)=b_{0}+b_{1} z^{-1}+\ldots+b_{m} z^{-m}
\end{aligned}
$$

$\{u(t)\}$ and $\{y(t)\}$ denote the plant input and output respectively. $\Delta=1-z^{-1}$ is the difference operator. Notice that the model (2.1) has the advantage that the controller will naturally contain an integrator (De Keyser $e t$ al. 1988).

The cost function has the following form

$$
J=\left\{\sum_{j=1}^{N_{1}}\left(y(t+j)-y_{r}(t+j)\right)^{2}+\lambda \sum_{j=1}^{N_{1}}(\Delta u(t+j-1))^{2}\right\}
$$

where $\left\{y_{r}(t)\right\}$ is a known reference sequence, $N_{1}$ is the prediction horizon whereas $\lambda$ is a weighting constant.

Using the following polynomial equations

$$
\begin{gathered}
1=F_{j}\left(z^{-1}\right) A\left(z^{-1}\right) \Delta+z^{-j} G_{j}\left(z^{-1}\right) \\
B\left(z^{-1}\right) F_{j}\left(z^{-1}\right)=E_{j}\left(z^{-1}\right)+z^{-j} H_{j}\left(z^{-1}\right)
\end{gathered}
$$

where $j=1,2, \ldots, N_{1}$ and

$$
\begin{aligned}
& F_{j}\left(z^{-1}\right)=f_{0}+f_{1} z^{-1}+\ldots+f_{j-1} z^{-j+1} \\
& G_{j}\left(z^{-1}\right)=g_{0}^{j}+g_{1}^{j} z^{-1}+\ldots+g_{n}^{j} z^{-n} \\
& E_{j}\left(z^{-1}\right)=e_{0}+e_{1} z^{-1}+\ldots+e_{j-1} z^{-j+1} \\
& H_{j}\left(z^{-1}\right)=h_{0}^{j}+h_{1}^{j} z^{-1}+\ldots+h_{m-1}^{j} z^{-m+1}
\end{aligned}
$$

the plant (2.1) can be written in the form

$$
y(t+j)=E_{j} \Delta u(t+j-1)+G_{j} y(t)+H_{j} \Delta u(t-1)
$$

where $j=1, \ldots, N_{1}$.

The equations (2.7) can be written in vector form as

$$
\mathbf{y}=\mathbf{E} u+\mathbf{G} y(t)+\mathbf{H} \Delta u(t-1)
$$

where

$$
\begin{aligned}
\mathbf{y}^{\mathbf{T}} & =\left[y(t+1), \ldots, y\left(t+N_{1}\right)\right] \\
\mathbf{u}^{\mathbf{T}} & =\left[\Delta u(t), \ldots, \Delta u\left(t+N_{1}-1\right)\right] \\
\mathbf{G}^{\mathbf{T}} & =\left[G_{1}, \ldots, G_{N_{1}}\right] \quad \mathbf{H}^{\mathbf{T}}=\left[H_{1}, \ldots, H_{N_{1}}\right]
\end{aligned}
$$


and where $\mathbf{E}$ is the $N_{1} \times N_{1}$ lower-triangular matrix

$$
\mathbf{E}=\left[\begin{array}{cccc}
e_{0} & & & \\
e_{1} & e_{0} & & \\
\ldots & & & \\
e_{N_{1}-1} & e_{N_{1}-2} & \ldots & e_{0}
\end{array}\right]
$$

Define

$$
\mathbf{y}_{r}^{\mathrm{T}}=\left[y_{r}(t+1), \ldots, y_{r}\left(t+N_{1}\right)\right]
$$

From the definition of $\mathbf{y}, \mathbf{y}_{\boldsymbol{r}}$ and $\mathbf{u}$ the cost function (2.2) can be written as

$$
J=\left\{\left(\mathbf{y}-\mathbf{y}_{r}\right)^{\mathrm{T}}\left(\mathbf{y}-\mathbf{y}_{r}\right)+\lambda \mathbf{u}^{\mathrm{T}} \mathbf{u}\right\}
$$

Substituting (2.8) into (2.10) and minimizing $J$ with respect to $\mathbf{u}$ results in the control law

$$
\mathbf{u}=\left(\mathbf{E}^{\mathrm{T}} \mathbf{E}+\lambda \mathbf{I}\right)^{-1} \mathbf{E}^{\mathrm{T}}\left[\mathbf{y}_{\mathbf{r}}-\mathbf{G} y(t)-\mathbf{H} \Delta u(t-1)\right]
$$

In order to avoid singularity of $\left(\mathbf{E}^{\mathbf{T}} \mathbf{E}+\lambda \mathbf{I}\right)$ and to reduce the computational load for large output horizons, the technique of imposing a control horizon $N_{u}\left(N_{u}<N_{1}\right)$ is used here, i.e., when $j \geqslant N_{u}$ we put $\Delta u(t+j)=0$. This implies a constant control after time instant $N_{u}$. The vector $\mathbf{u}$ and matrix $\mathbf{E}$ in (2.9) then take the forms, respectively,

$$
\begin{gathered}
\mathbf{u}^{\mathbf{T}}=\left[\Delta u(t), \ldots, \Delta u\left(t+N_{u}-1\right)\right] \\
\mathbf{E}=\left[\begin{array}{cccc}
e_{0} & & \\
e_{1} & e_{0} & & \\
\cdots & & & \\
e_{N_{u}-1} & e_{N_{u}-2} & \cdots & e_{0} \\
\cdots & & & \\
e_{N_{1}-1} & e_{N_{1}-2} & \cdots & e_{N_{1}-N_{u}}
\end{array}\right]
\end{gathered}
$$

where the dimension of $\mathbf{E}$ now becomes $N_{1} \times N_{u}$

Writing the first row of $\left(\mathbf{E}^{\mathrm{T}} \mathbf{E}+\lambda \mathbf{I}\right)^{-\mathbf{1}} \mathbf{E}^{\mathrm{T}}$ as

$$
\mathbf{P}^{\mathrm{T}}=\left[p_{1}, \ldots, p_{N_{1}}\right]
$$

we define

$$
P\left(z^{-1}\right)=p_{N_{1}}+p_{N_{1}-1} z^{-1}+\ldots+p_{1} z^{-N_{1}+1}
$$

Then, from (2.11) and the receding horizon strategy (Clarke et al. 1987), the control law is given by

$$
\begin{aligned}
\Delta u(t) & =\mathbf{P}^{\mathrm{T}}\left[y_{r}-\mathbf{G} y(t)-\mathbf{H} \Delta u(t-1)\right] \\
& =P\left(z^{-1}\right) y_{r}\left(t+N_{1}\right)-\alpha\left(\mathrm{z}^{-1}\right) y(t)-\beta\left(z^{-1}\right) \Delta u(t-1)
\end{aligned}
$$

where

$$
\alpha\left(z^{-1}\right)=\sum_{j=1}^{N_{1}} p_{j} G_{j}\left(z^{-1}\right) \quad \beta\left(z^{-1}\right)=\sum_{j=1}^{N_{1}} p_{j} H_{j}\left(z^{-1}\right)
$$

and where the degrees of $\alpha\left(z^{-1}\right)$ and $\beta\left(z^{-1}\right)$ are $n$ and $m-1$ respectively. 


\section{A direct adaptive algorithm}

It is well known that, in the direct version of adaptive control, one has to rearrange a model so that it is expressed directly in terms of the control law parameters. Thus, in effect, one directly estimates the control law parameters. In this section an implicit model is first derived, then a direct adaptive algorithm is suggested.

\subsection{Implicit model}

Let us denote the first row of $\left(\mathbf{E}^{\mathrm{T}} \mathbf{E}+\lambda \mathbf{I}\right)^{-1}$ by

$$
\mathbf{Q}^{\mathrm{T}}=\left[q_{1}, \ldots, q_{N_{\mathrm{u}}}\right]
$$

and define

$$
Q\left(z^{-1}\right)=q_{N_{u}}+q_{N_{u}-1} z^{-1}+\ldots+q_{1} z^{-N_{u}+1}
$$

Multiplying by $\mathbf{E}^{\mathrm{T}}$ and adding $\lambda \mathbf{I} u$ on both sides of (2.8) we have

$$
\mathbf{E}^{\mathrm{T}} \mathbf{y}+\lambda \mathbf{I} \mathbf{u}=\mathbf{E}^{\mathrm{T}} \mathbf{E u}+\lambda \mathbf{I} \mathbf{u}+\mathbf{E}^{\mathrm{T}}(\mathbf{G} y(t)+\mathbf{H} \Delta u(t-1))
$$

which results in

$$
\mathbf{u}=\left(\mathbf{E}^{\mathrm{T}} \mathbf{E}+\lambda \mathbf{I}\right)^{-1}\left[\mathbf{E}^{\mathrm{T}}(\mathbf{y}-\mathbf{G} y(t)-\mathbf{H} \Delta u(t-1))+\lambda \mathbf{I} \mathbf{u}\right]
$$

Using (2.14), (3.2), (2.16) and (2.17) the first row of (3.4) can be written as

$$
\begin{aligned}
\Delta u(t)= & P\left(z^{-1}\right) y\left(t+N_{1}\right)+\lambda Q\left(z^{-1}\right) \Delta u\left(t+N_{u}-1\right) \\
& -\alpha\left(z^{-1}\right) y(t)-\beta\left(z^{-1}\right) \Delta u(t-1)
\end{aligned}
$$

Define

$$
\phi\left(t+N_{1}\right)=P\left(z^{-1}\right) y\left(t+N_{1}\right)+\lambda Q\left(z^{-1}\right) \Delta u\left(t+N_{u}-1\right)
$$

Then (3.5) can be written as

$$
\begin{aligned}
\phi\left(t+N_{1}\right) & =\alpha\left(\mathrm{z}^{-1}\right) y(t)+\beta\left(z^{-1}\right) \Delta u(t-1)+\Delta u(t) \\
& =\mathbf{X}(t)^{\mathbf{T}} \boldsymbol{\theta}
\end{aligned}
$$

where

$$
\begin{aligned}
\mathbf{X}(t)^{\mathrm{T}} & =[y(t), \ldots, y(t-n), \Delta u(t), \ldots, \Delta u(t-m)] \\
\boldsymbol{\theta}^{\mathrm{T}} & =\left[\alpha_{0}, \alpha_{1}, \ldots, \alpha_{u}, 1, \beta_{0}, \ldots, \beta_{m-1}\right]
\end{aligned}
$$

We know from (2.15) that equation (3.7) is expressed directly in terms of the control law parameters. If we choose $u(t)$ to satisfy

$$
P\left(z^{-1}\right) y_{r}\left(t+N_{1}\right)=X(t)^{\mathrm{T}} \theta
$$

it is evident that (3.8) is equal to the control law (2.15).

\subsection{Direct adaptive algorithm}

In the following direct adaptive GPC algorithm (3.7) is taken as a parameter estimation equation, which implies that $\phi(t)$ should be known at time $t$. From (2.6), (2.12)-(2.14), (3.1) and (3.2) it is clear that if $e_{0}, e_{1}, \ldots, e_{N_{1}-1}$ are known and $\lambda$ is given, the coefficients of $P\left(z^{-1}\right)$ and $Q\left(z^{-1}\right)$ can be computed off-line, and $\phi(t)$ will be available at time $t$. We know from (2.3) and (2.4) that the coefficients of $E_{N}\left(z^{-1}\right)$, i.e. $e_{0}, e_{1}, \ldots, e_{N_{1}-1}$ 
are simply the first $N_{1}$ terms of the plant's step response. Therefore, under the assumptions

A1: The polynomial degrees $n$ and $m$ are known.

A2: The first $N_{1}$ coefficients of the plant's step response, i.e. $e_{0}, e_{1}, \ldots, e_{N_{1}-1}$ are known.

we have the following adaptive algorithm:

Data: Given the prediction horizon $N_{1}$, the control horizon $N_{w}$, the weighting constant $\lambda$ and the first $N_{1}$ coefficients of the plant step response, i.e. $e_{0}, e_{1}, \ldots, e_{N_{1}-1}$.

(a) Compute $\phi(t)$ from

$$
\phi(t)=P\left(z^{-1}\right) y(t)+\lambda Q\left(z^{-1}\right) \Delta u\left(t+N_{u}-N_{1}-1\right)
$$

(b) Compute $\hat{\theta}(t)$ using the following estimation algorithm given in Goodwin, Leal, Mayne and Middleton (1986).

$$
\hat{\theta}(t)=\hat{\theta}(t-1)+\frac{\rho \mathbf{X}\left(t-N_{1}\right)\left(\phi(t)-\mathbf{X}\left(t-N_{1}\right)^{\mathrm{T}} \hat{\theta}(t-1)\right)}{1+\mathbf{X}\left(t-N_{1}\right)^{\mathrm{T}} \mathbf{X}\left(t-N_{1}\right)}
$$

where $0<\rho<2$.

(c) Compute the control $u(t)$ from

$$
P\left(z^{-1}\right) y_{\mathrm{r}}\left(t+N_{1}\right)=\mathbf{X}(t)^{\mathrm{T}} \theta(t)
$$

(d) Increase $t$ by one and return to $(a)$.

In comparison with the algorithm given in Ortega and Sanchez (1989), our algorithm uses only one parameter estimator at each sampling instant, whereas a bank of interlaced estimators is used in Ortega and Sanchez (1989). The on-line computational load in the latter algorithm is therefore quite heavy.

\subsection{Convergence analysis}

The following assumption is, in addition to $\mathrm{A} 1$ and $\mathrm{A} 2$, made for the global convergence analysis of our algorithm.

A3: The characteristic polynomial

$$
T\left(z^{-1}\right)=P\left(z^{-1}\right) B\left(z^{-1}\right)+z^{-N_{1}+N_{u}} \lambda Q\left(z^{-1}\right) A\left(z^{-1}\right) \Delta
$$

is stable, i.e. $T(q) \neq 0$ for $|q|>1$.

The assumption A3 means that when the plant's parameters are known, the control law (3.11) with suitable choices of $N_{1}, N_{u}, \lambda$ and $e_{j}\left(j=0, \ldots, N_{1}-1\right)$ stabilizes the plant.

Theorem 3.1. Under assumptions A1-A3, the direct adaptive algorithm given above leads to

S1) $\{y(t)\}$ and $\{\Delta u(t)\}$ are bounded sequences.

S2) $\lim _{t \rightarrow \infty}\left|P\left(y(t)-y_{r}(t)\right)+\lambda Q \Delta u\left(t+N_{u}-N_{1}-1\right)\right|=0$ 
Proof: Define, respectively, the estimation error $\varepsilon(t)$ and the generalized tracking error $e(t)$ as

$$
\begin{aligned}
& \varepsilon(t)=\phi(t)-\mathbf{X}\left(t-N_{1}\right)^{\mathrm{T}} \hat{\theta}(t-1) \\
& e(t)=P\left(y(t)-y_{r}(t)\right)+\lambda Q \Delta u\left(t+N_{u}-N_{1}-1\right)
\end{aligned}
$$

Multiplying (2.1) by $P\left(z^{-1}\right),(3.14)$ by $\Delta A\left(z^{-1}\right)$, and adding together we obtain

$$
T\left(z^{-1}\right) \Delta u(t-1)=\Delta A\left(z^{-1}\right) e(t)+\Delta A\left(z^{-1}\right) P\left(z^{-1}\right) y_{r}(t)
$$

Multiplying (2.1) by $\lambda Q\left(z^{-1}\right),(3.13)$ by $B\left(z^{-1}\right)$, and adding together we obtain

$$
T\left(z^{-1}\right) y(t)=B\left(z^{-1}\right) e(t)+B\left(z^{-1}\right) P\left(z^{-1}\right) y_{r}(t)
$$

Using assumption A2, the boundedness of $\left\{y_{r}(t)\right\},(3.14),(3 \cdot 15)$ and lemma B.3.3 in Goodwin and Sin (1984) we have

$$
\begin{gathered}
\left|y\left(t-N_{1}\right)\right| \leqslant K_{1}+K_{2} \max _{0 \leqq \mathrm{~T} \leqq t}|e(\tau)| \\
\left|\Delta u\left(t-N_{1}\right)\right| \leqslant K_{3}+K_{4} \max _{0 \leqq \mathrm{~T} \leqq t}|e(\tau)|
\end{gathered}
$$

From the definition of $\mathbf{X}(t),(3.16)$ and (3.17) we have

$$
\left\|\mathbf{X}\left(t-N_{1}\right)\right\| \leqslant K_{5}+K_{6} \max _{0 \leqq \mathrm{~T} \leqq t}|e(\tau)|
$$

where $K_{1}, \ldots, K_{6}$ are positive constants.

Substituting (3.11) and (3.12) into (3.13) yields

$$
e(t)=\varepsilon(t)+\mathbf{X}\left(t-N_{1}\right)^{\mathrm{T}}\left(\hat{\theta}(t-1)-\hat{\theta}\left(t-N_{1}\right)\right)
$$

From (3.19) and lemma 3.3.2 ((a) and (e)) in Goodwin and Sin (1984) we have

$$
\lim _{t \rightarrow \infty} \frac{e^{2}(t)}{1+\mathbf{X}\left(t-N_{1}\right)^{\mathbf{T}} \mathbf{X}\left(t-N_{1}\right)}=0
$$

Using (3.18), (3.20) and the key technical lemma in Goodwin and Sin (1984), the results S1 and S2 follow immediately.

\section{An improved direct adaptive algorithm}

The adaptive algorithm given in the last section is very attractive because of its simplicity. The weakness of the algorithm is the requirement for a priori knowledge of the first $N_{1}$ terms of the plant's step response. In most cases, however, these terms are hardly known exactly. A heuristic approach is simply to use their estimates. In this section we will propose an improved direct adaptive algorithm which is based upon that. 


\subsection{Normalized model}

Assume $\bar{e}_{j}, j=0, \ldots, N_{1}-1$ to be estimates of the first $N_{1}$ terms of the plant's step response and define

$$
\overline{\mathbf{E}}=\left[\begin{array}{cccc}
\bar{e}_{0} & & & \\
\bar{e}_{1} & \bar{e}_{0} & & \\
\ldots & & & \\
\bar{e}_{N_{u}-1} & \bar{e}_{N_{u}-2} & \ldots & \bar{e}_{0} \\
\ldots & & & \\
\bar{e}_{N_{1}-1} & \bar{e}_{N_{1}-2} & \ldots & \bar{e}_{N_{1}-N_{u}}
\end{array}\right]
$$

Furthermore, write the first rows of $\left(\overline{\mathbf{E}}^{\mathrm{T}} \overline{\mathbf{E}}+\lambda \mathbf{I}\right)^{-1} \overline{\mathbf{E}}^{\mathrm{T}}$ and $\left(\overline{\mathbf{E}}^{\mathrm{T}} \overline{\mathbf{E}}+\lambda \mathbf{I}\right)^{-1}$ as, respectively,

$$
\begin{aligned}
& \overline{\mathbf{P}}^{\mathrm{T}}=\left[\bar{p}_{1}, \ldots, \bar{p}_{N_{1}}\right] \\
& \overline{\mathbf{Q}}^{\mathrm{T}}=\left[\bar{q}_{1}, \ldots, \bar{q}_{N_{u}}\right]
\end{aligned}
$$

and define

$$
\begin{aligned}
& \bar{P}\left(z^{-1}\right)=\bar{p}_{N_{1}}+\bar{p}_{N_{1}-1} z^{-1}+\ldots+\bar{p}_{1} z^{-N_{1}+1} \\
& \bar{Q}\left(z^{-1}\right)=\bar{q}_{N_{u}}+\bar{q}_{N_{u}-1} z^{-1}+\ldots+\bar{q}_{1} z^{-N_{u}+1}
\end{aligned}
$$

Using (4.4) and (4.5), equation (3.7) can be written as

$$
\bar{\phi}(t)=\mathbf{X}\left(t-N_{1}\right)^{\mathrm{T}} \theta+\delta(t)
$$

where

$$
\begin{aligned}
& \bar{\phi}(t)=\bar{P} y(t)+\lambda \bar{Q} \Delta u\left(t+N_{u}-N_{1}-1\right) \\
& \delta(t)=(\bar{P}-P) y(t)+\lambda(\bar{Q}-Q) \Delta u\left(t+N_{u}-N_{1}-1\right)
\end{aligned}
$$

The term $\delta(t)$ in (4.6) may be interpreted as unmodeled dynamics. Obviously, $\delta(t)$ may be unbounded since $\delta(t)$ involves $y(t)$ and $\Delta u(t)$. The normalization technique suggested in Cluett, Shah and Fisher (1987) and Cluett, Martin-Sanchez, Shah and Fisher (1988) is used here to guarantee the boundedness of the unmodeled dynamics. Define

$$
n(t)=\max \left(\max _{i \leqq i \leqq d}\left|\psi_{i}(t)\right|, C\right)
$$

where

$$
\psi(t)^{\mathbf{T}}=\left[y(t), \ldots, y\left(t-n-N_{1}\right), \Delta u(t-1), \ldots, \Delta u\left(t-m-N_{1}\right)\right]
$$

and $d$ is the dimension of $\psi(t)$. Let $\psi_{i}(t)$ denote the $i$ th element of $\psi(t)$. $C$ is any positive constant and is used to prevent division by zero.

Using the following normalized variables

$$
\begin{aligned}
\phi^{n}(t) & =\bar{\phi}(t) / n(t) \\
\mathbf{X}^{n}\left(t-N_{1}\right) & =\mathbf{X}\left(t-N_{1}\right) / n(t) \\
\delta^{n}(t) & =\delta(t) / n(t)
\end{aligned}
$$

equation (4.6) takes the form

$$
\bar{\phi}^{n}(t)=\mathbf{X}^{n}\left(t-N_{1}\right)^{\mathrm{T}} \theta+\delta^{n}(t)
$$


It is easy to show that both $\delta^{n}(t)$ and $\mathbf{X}^{n}\left(t-N_{1}\right)$ defined by respectively (4.12) and (4.13) are bounded. Let $M$ be a known upper bound of $\left|\delta^{n}(t)\right|$, i.e.

$$
\left|\delta^{n}(t)\right| \leqslant M . \quad \forall t \geqslant 0
$$

\subsection{Improved direct adaptive algorithm}

The improved algorithm and our global convergence analysis of it are based on the following assumptions:

B1. The polynomial degrees $n$ and $m$ are known.

B2. $M$ is known.

B3. The characteristic polynomial

$$
\bar{T}\left(z^{-1}\right) \bar{P}\left(z^{-1}\right)+B\left(z^{-1}\right) z^{-N_{1}+N_{u}} \lambda \bar{Q}\left(z^{-1}\right) A\left(z^{-1}\right) \Delta
$$

is stable, i.e. $\bar{T}(q) \neq 0$ for $|q|>1$.

Define the estimation error $\bar{\varepsilon}(t)$ as

$$
\bar{\varepsilon}(t)=\bar{\phi}(t)-\mathbf{X}\left(t-N_{1}\right)^{\mathrm{T}} \hat{\theta}(t-1)
$$

Dividing both sides on (4.16) by $n(t)$, the normalized $\bar{\varepsilon}(t)$ can be written as

$$
\bar{\varepsilon}^{n}(t)=\bar{\phi}^{n}(t)-\mathbf{X}^{n}\left(t-N_{1}\right)^{\mathrm{T}} \hat{\theta}(t-1)
$$

In order to handle the unmodeled dynamics $\delta^{n}(t)$, the recursive parameter estimation scheme with a dead zone given in Goodwin et al. (1986) is used for the normalized model (4.14), viz.

$$
\hat{\theta}(t)=\hat{\theta}(t-1)+\frac{\rho \mathbf{X}^{n}\left(t-N_{1}\right) f\left(M, \bar{\varepsilon}^{n}(t)\right)}{1+\mathbf{X}^{n}\left(t-N_{1}\right)^{\mathbf{T}} \mathbf{X}^{n}\left(t-N_{1}\right)}
$$

where

$$
f\left(M, \bar{\varepsilon}^{n}(t)\right)=\left\{\begin{array}{cc}
\bar{\varepsilon}^{n}(t)-M & \text { if } \bar{\varepsilon}^{n}(t)>M \\
0 & \text { if }\left|\bar{\varepsilon}^{n}(t)\right| \leqslant M \\
\bar{\varepsilon}^{n}(t)+M & \text { if }\left|\bar{\varepsilon}^{n}(t)\right| \leqslant M
\end{array}\right.
$$

and where $0<\rho<1$.

The above considerations motivate the following improved direct adaptive algorithm.

Data: Given $N_{1}, N_{u}, \lambda$ and the estimates of the first $N_{1}$ terms of the plant's step response, i.e. $\bar{e}_{0}, \bar{e}_{1}, \ldots, \bar{e}_{N_{1}-1}$.

(a) Compute $\phi(t)$ from

$$
\bar{\phi}(t)=\bar{P}\left(z^{-1}\right) y(t)+\lambda \bar{Q}\left(z^{-1}\right) \Delta u\left(t+N_{u}-N_{1}-1\right)
$$

(b) Compute $\hat{\theta}(t)$ using the estimation scheme (4.18).

(c) Compute the control $u(t)$ from

$$
\bar{P}\left(z^{-1}\right) y_{r}\left(t+N_{1}\right)=X(t)^{\mathrm{T}} \hat{\theta}(t)
$$

(d) Increase $t$ by one and return to (a). 


\subsection{Convergence analysis}

Lemma 4.1. The parameter estimation scheme (4.18) has the following properties:

(ii)

$$
\begin{aligned}
\lim _{t \rightarrow \infty} \frac{f\left(M, \bar{\varepsilon}^{n}(t)\right)}{1+\mathbf{X}^{n}\left(t-N_{1}\right)^{\mathrm{T}} \mathbf{X}^{n}\left(t-N_{1}\right)} & =0 \\
\lim _{t \rightarrow \infty}\|\hat{\theta}(t)-\theta(t-k)\| & =0
\end{aligned}
$$

Proof: see Goodwin et al. (1986).

Define the generalized tracking error $\bar{e}(t)$ as

$$
\bar{e}(t)=\bar{P}\left(y(t)-y_{r}(t)\right)+\lambda \bar{Q} \Delta u\left(t+N_{u}-N_{1}-1\right)
$$

Lemma 4.2. Subject to assumptions B1-B3, there exist positive constants $C_{1}$ and $C_{2}$ such that

$$
C_{1}\|\psi(t)\| \leqslant \max _{0 \leqq \tau \leqq t}|\bar{e}(\tau)|+C_{2}
$$

Proof: Multiplying (2.1) by $\bar{P}\left(z^{-1}\right),(4.22)$ by $\Delta A\left(z^{-1}\right)$, and adding together we obtain

$$
\bar{T}\left(z^{-1}\right) \Delta u(t-1)=\Delta A\left(z^{-1}\right) \bar{e}(t)+\Delta A\left(z^{-1}\right) \bar{P}\left(z^{-1}\right) y_{r}(t)
$$

Multiplying (2.1) by $\lambda \bar{Q}\left(z^{-1}\right),(4.22)$ by $B\left(z^{-1}\right)$, and adding together we obtain

$$
\bar{T}\left(z^{-1}\right) y(t)=B\left(z^{-1}\right) \bar{e}(t)+B\left(z^{-1}\right) \bar{P}\left(z^{-1}\right) y_{r}(t)
$$

Using assumption B3, the boundedness of $y_{r}(t),(4.24),(4.25)$ and lemma B.3.3 in Goodwin and Sin (1984) we have

$$
\begin{array}{r}
|y(t)| \leqslant K_{1}^{\prime}+K_{2}^{\prime} \max _{0 \leqq r \leqq t}|\bar{e}(\tau)| \\
|\Delta u(t-1)| \leqslant K_{3}^{\prime}+K_{4}^{\prime} \max _{0 \leqq t \leqq t}|\bar{e}(\tau)|
\end{array}
$$

From (4.10), (4.26) and (4.27) it follows that

$$
\|\psi(t)\| \leqslant K_{5}^{\prime}+K_{6}^{\prime} \max _{0 \leqq t \leqq t}|\bar{e}(\tau)|
$$

where $K_{1}^{\prime}, \ldots, K_{6}^{\prime}$ are positive constants. Thus (4.23) follows immediately from (4.28) with $C_{1}=1 / \mathrm{K}_{6}^{\prime}$ and $C_{2}=K_{5}^{\prime} / K_{6}^{\prime}$.

Theorem 4.1. Under assumptions B1-B3, if there exists a constant $C_{1}$ which satisfies

B4: $C_{1}>M$

then the direct adaptive algorithm given in this section leads to

S1) $\{y(t)\}$ and $\{\Delta u(t)\}$ are bounded sequences.

S2) $\lim _{t \rightarrow \infty}\left|\left[\bar{P}\left(y(t)-y_{r}(t)\right)+\lambda \bar{Q} \Delta u\left(t+N_{u}-N_{1}-1\right)\right] / n(t)\right| \leqslant M$

Proof: Since $\mathbf{X}^{n}\left(t-N_{1}\right)$ is bounded we know from lemma 4.1 (i) that

$$
\lim _{t \rightarrow \infty} f\left(M, \bar{\varepsilon}^{n}(t)\right)=0
$$


Substituting (4.16) and (4.21) into (4.22) yields

$$
\bar{e}(t)=\bar{\varepsilon}(t)+\mathbf{X}\left(t-N_{1}\right)^{\mathrm{T}}\left(\hat{\theta}(t-1)-\hat{\theta}\left(t-N_{1}\right)\right)
$$

Dividing both sides by $n(t)$ yields

$$
\bar{e}^{n}(t)=\bar{\varepsilon}^{n}(t)+\mathbf{X}^{n}\left(t-N_{1}\right)^{\mathrm{T}}\left(\hat{\theta}(t-1)-\hat{\theta}\left(t-N_{1}\right)\right)
$$

Using Lemma 4.1 (ii), (4.19), (4.29) and (4.31) we have

$$
\lim _{t \rightarrow \infty} f\left(M, \bar{e}^{n}(t)\right)=0
$$

which from (4.19) means that

$$
\lim _{t \rightarrow \infty}\left|\bar{e}^{n}(t)\right| \leqslant M
$$

From B4, (4.9) and (4.33) we have

$$
\begin{aligned}
C_{1}>M & \geqslant \lim _{t \rightarrow \infty} \frac{|\bar{e}(t)|}{n(t)} \\
& =\lim _{t \rightarrow \infty} \frac{|\bar{e}(t)|}{\max \left(\max _{1 \leqq i \leqq d}\left|\psi_{i}(t)\right|, C\right)} \\
& \geqslant \lim _{t \rightarrow \infty} \frac{|\bar{e}(t)|}{\max (\|\psi(t)\|, C)}
\end{aligned}
$$

If $\{\|\psi(t)\|\}$ is bounded, then $\mathrm{S} 1$ follows immediately. Therefore, assume the sequence $\{\|\psi(t)\|\}$ to be unbounded. From lemma 4.2 there exists a subsequence $\left\{t_{n}\right\}$ such that

where

$$
C_{1}\left\|\psi\left(t_{n}\right)\right\| \leqslant\left|\bar{e}\left(t_{n}\right)\right|+C_{2}
$$

$$
\left|\bar{e}\left(t_{n}\right)\right|=\max _{0 \leqq \imath \leqq 1}|\bar{e}(\tau)|
$$

Dividing both sides of (4.35) by $\left\|\psi\left(t_{n}\right)\right\|$ we obtain

$$
C_{1} \leqslant \frac{\left|\bar{e}\left(t_{n}\right)\right|}{\left\|\psi\left(t_{n}\right)\right\|}+\frac{C_{2}}{\left\|\psi\left(t_{n}\right)\right\|}
$$

Since $\left\|\psi\left(t_{n}\right)\right\| \rightarrow \infty$ as $t_{n} \rightarrow \infty$, the last term on the right hand side of (4.36) tends to zero which implies that (4.36) will violate inequality (4.34). Therefore an unbounded sequence $\{\|\psi(t)\|\}$ cannot exist and S1 must hold. From (4.33) and (4.22) S2 follows immediately.

From theorem 4.1 we observe that the improved algorithm guarantees stability of the closed-loop system and makes the generalized error $\bar{e}^{n}(t)$ bounded. From (4.8) it is obvious that the unmodeled dynamics $\delta(t)$ comes from mismatch between $e_{j}$ and $\bar{e}_{j}$. If this mismatch is smaller, $\delta(t)$ will also be smaller, and the required upper bound $M$ may be decreased. In the matched case $(\delta(t)=0)$, the generalized error converges asymptotically to zero. This illustrates the link between the first algorithm and the improved one. 


\section{Conclusion}

In this paper a direct adaptive GPC algorithm and an improved version have been proposed. Global convergence of the algorithms have been analysed by using standard analysis techniques under some reasonable assumptions. We have, for the sake of simplicity, used a projection estimation scheme in our algorithms. Similar results can be obtained for corresponding least squares schemes. The combination of the normalization technique and the parameter estimation scheme with a dead zone makes the global convergence analysis of the improved algorithm quite simple. The results presented in this paper are conceptually important in that they fill a gap existing in previous published papers on the direct version of adaptive GPC algorithms.

\section{REFERENCES}

Clarke, D. W., Mohtadi, C. and TuFfs, P. S. (1987). Generalized predictive control-Part I and II, Automatica, 23, 137-160.

Cluett, W. R., Martin-Sanchez, J. M., Shah, S. L. and Fisher, D. G. (1988). Stable discretetime adaptive control in the presence of unmodeled dynamics, IEEE Trans., AC 33, $410-414$.

Cluett, W. R., Shah, S. L., and Fisher, D. G. (1987). Stable robust adaptive control in the presence of unmodeled dynamics. Int. J. Control, 45, 1265-1273.

Goodwin, G. C., LEAL, R. L., MAYNE, D. Q., and MidDleTON, R. H. (1986). Rapprochement between continuous and discrete model reference adaptive control, Automatica, 22, 199-207.

Goodwin, G. C. and SIN, K. S. (1984), Adaptive filtering, prediction and control (Englewood Cliffs, NJ: Prentice Hall).

de Keyser, R. M. C., VAN de Velde Ph. G. A. and Dumortier, F. A. G. (1988). A comparative study of self-adaptive long-range predictive control methods. Automatica, 24, 149-163.

Kramer, K. and Unbehauen, H. (1988). Survey to adaptive long-range predictive control, in Proc. 12th IMACS World Congress on Scientific Computation, Paris, 1988.

LELIC, M. A. and ZARROP, M. B. (1987). Generalized pole placement self-tuning controller-Part I and II. Int. J. Control, 46, 548-568.

OrtegA, R. and SANCHEZ, G. (1989). Globally convergent multistep receding horizon adaptive controller. Int. J. Control, vol. 49, pp. 1655-1664.

YDSTIE, B. E. (1984). Extended horizon adaptive control, in Proc. IF AC 9th World Congress, Budapest, Hungary, 1984. 